\section{Deciphering the rules of microRNA targeting}

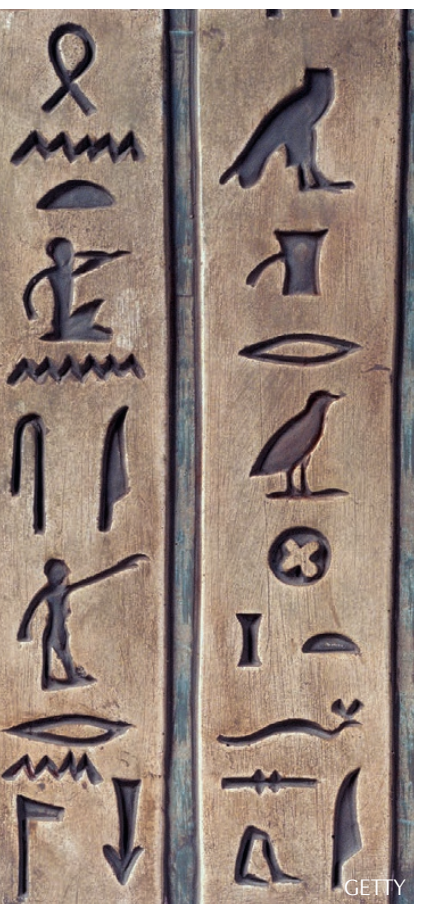

MicroRNAs (miRNAs) are thought to directly regulate over $60 \%$ of human mRNAs and have been found to be involved in numerous biological processes as well as diseases. Nonetheless, the 'rules' by which they identify their targets are still not fully understood. Kim et al. set out to systematically describe the distinct types of functional interactions between miRNAs and their targets.

Canonical site types are 6- to 7-nucleotide sites of perfect complementarity between an miRNA and its target mRNA. Large-scale studies have highlighted their importance; however, other approaches have revealed non-canonical site types of unknown functionality. The team started by defining a general 'site type', representing a collection of the interactions between each nucleotide from the miRNA and the interacting nucleotide on the mRNA. By compiling the potential site types between 177 conserved human miRNAs and 18,625 mRNAs, they identified a potential four trillion unique site types. By limiting the results to site types present in more than eight target sites, this number was reduced to two billion site types.

Utilizing microarray data of gene expression, the group identified seven new non-canonical site types including those referred to as the 'context-dependent non-canonical site type'. The efficacy of these site types was dependent on local context of each target site, including local AU content near the target site, and stability of the miRNA-mRNA pairing.

The authors went on to validate the functionality of the new site types with luciferase assays and transcriptome-wide data from human cells, mice and zebrafish. Intriguingly, evolutionary analysis indicated that these non-canonical site types and context-dependent non-canonical site types are under purifying selection relative to control sites. Although the group notes that these site types are weaker in their ability to downregulate mRNAs compared with canonical site types, the data point towards a biological importance of these non-canonical site types.

By undertaking a massive computational analysis of miRNA site type identification, the authors have detected previously unknown non-canonical sites that are likely to have a biological role. Although Kim et al. add a caveat to their findings by pointing out the use of microarray data and the potential risk of excluding non-canonical site types that exhibit weaker efficacies than their statistical thresholds, the work helps to unravel the rules by which miRNAs function.

Ross Cloney, Associate Editor, Nature Communications

ORIGINAL ARTICLE Kim, D. et al. General rules for functional microRNA targeting. Nat. Genet http://dx.doi.org/10.1038/ng.3694 (2016) FURTHER READING Hausser, J. \& Zavolan, M. Identification and consequences of miRNA-target interactions - beyond repression of gene expression. Nat. Rev. Genet. 15, 599-612 (2014) 